\title{
Statistical Properties of the Squeezed Displaced Number States
}

\author{
Célia M. A. Dantas, Norton G. de Almeida and B. Baseia \\ Instituto de Física, Universidade Federal de Goiás, \\ Caixa Postal 131, 74001-970, Goiânia, GO, Brazil
}

Received 1 August, 1998. Revised version received 5 October, 1998

\begin{abstract}
Squeezed Displaced Number States of the light were introduced in the recent literature. They exhibit various nonclassical properties as sub-Poissonian statistics, squeezing and oscillations in the photon-number distribution. Here we investigate other properties of these fields, as waiting-time and photoelectron-counting distributions. We considerably simplify previous calculations in the literature while showing that these states constitute a unified approach for number, coherent, and squeezed states.
\end{abstract}

PACS Numbers: 42.50. -p 42.50.Dv 03.65.-w

\section{Introduction}

In recent years, many authors have investigated new quantum states of the electromagnetic field. The squeezed state can be considered as one of the most studied state [1]. It is characterized by the fact that the indetermination in one of the two quadraturecomponents of the electromagnetic field is smaller than in the usual coherent states. It is of great importance, as in optical communication and interferometry [2]. Special attention is given to three classes of squeezed states: (i) squeezed vacuum states, (ii) squeezed coherent states and (iii) squeezed number states.

Here we will study a new class of squeezed states, called Squeezed Displaced Number State [SDNS], recently introduced in the literature [3]. We show that this class contains, as particular cases, various states already studied in the literature, such as the squeezed coherent states [4], squeezed number states [5], displaced number states [6], including the coherent state and number states. The photoelectron-counting distribution $P(m, T)$ and the distribution of waiting-time intervals $\omega(\tau)$, between the arrivals of successive photons, are analyzed as a function of various parameters. Depending on the values of the parameters characteriz- ing a particular realization of the SDNS, it may exhibit sub-Poissonian or super-Poissonian statistics, which is analyzed in terms of Mandel's $Q$-parameter. As a consequence, it exhibits photon bunching or photon antibunching, which is analyzed in terms of the distribution $\omega(\tau)$.

This paper is organized as follows: In Section II we introduce the SDNS and analyze the photon number distribution $P(j)$, including variances of number operator $\hat{n}=\hat{a}^{+} \hat{a}$ and quadrature operators $\hat{X}_{1}=\left(\hat{a}+\hat{a}^{\dagger}\right) / 2$ and $\hat{X}_{2}=\left(\hat{a}-\hat{a}^{\dagger}\right) / 2 i$, where $\hat{a}^{\dagger}(\hat{a})$ is the creation (annihilation) operator for photons in a single-mode state. In Section III we analyze the photoelectron-counting distribution $P(m, T)$ and in Section IV we discuss the behavior of waiting-time distribution $\omega(\tau)$. Finally, Section $\mathrm{V}$ contains a summary of results and conclusion.

\section{Squeezed Displaced Number State}

The squeezed displaced number state $|n, \alpha, z\rangle$ is obtained by the application of the squeeze operator $\widehat{S}(z)$ followed by the displaced operator $\widehat{D}(\alpha)$, on the number state $|n\rangle$,

\footnotetext{
*E-mail: cdantas@fis.ufg.br
} 


$$
|n, \alpha, z\rangle \equiv \widehat{D}(\alpha) \widehat{S}(z)|n\rangle,
$$

where $\widehat{D}(\alpha)$ and $\widehat{S}(z)$ are the displacement and squeeze operators, given respectively by

$$
\begin{gathered}
\widehat{D}(\alpha)=\exp \left(\alpha \hat{a}^{+}-\alpha^{*} \hat{a}\right), \\
\widehat{S}(z)=\exp \left[\left(z \hat{a}^{+2}-z^{*} \hat{a}^{2}\right) / 2\right],
\end{gathered}
$$

where $\alpha$ is the coherence parameter, $\alpha=|\alpha| e^{i \theta}$, and $z$ is the squeeze parameter, $z=r e^{i \phi}$.

Alternatively, the equation (2.1) can be written as

$$
|n, z, \beta\rangle=\widehat{S}(z) \hat{D}(\beta)|n\rangle
$$

with

$$
\beta=\mu \alpha+\nu \alpha^{*}
$$

where $\mu=\cosh (r)$ and $\nu=e^{i \phi} \sinh (r)$. The Eqs.(2.1)-(2.4) are connected, via the unitary trans- formation, $\widehat{D}(\beta)=\widehat{S}^{+}(z) \widehat{D}(\alpha) \widehat{S}(z)$ with $\widehat{S}^{+}(z) \widehat{S}(z)=$ $\widehat{S}(z) \widehat{S}^{+}(z)=1$.

The coefficients $C_{j}$ for the SDNS in the Fock representation are defined as

$$
C_{j} \equiv\langle j|\widehat{S}(z) \widehat{D}(\beta)| n\rangle=\langle j \mid n, z, \beta\rangle
$$

and the application of the completeness relation of the number states $\{|m\rangle\}$, in the Eq.(2.6), gives

$$
\begin{gathered}
C_{j}=\sum_{m=0}^{\infty}\langle j|\widehat{S}(z)| m\rangle\langle m|\widehat{D}(\beta)| n\rangle \\
=\sum_{m=0}^{\infty} S_{j m} \cdot D_{m n},
\end{gathered}
$$

where $S_{j m}, D_{m n}$ are respectively the coefficients of the squeezed number state [5] (with $\phi=0$ ) and the displaced number state [6], given by

$$
S_{j m}=\frac{\sqrt{m ! j !}}{(\cosh r)^{j+1 / 2}} \cdot\left(\frac{1}{2} \tanh r\right)^{(m-j) / 2} F(r, m, j) \cdot \cos ^{2}(j-m) \frac{\pi}{2}
$$

with

$$
F(r, m, j)=\sum_{k=\frac{1}{2}(j-m)}^{\frac{1}{2} j} \frac{(-1)^{k}\left(2^{-1} \sinh r\right)^{2 k}}{k !(j-2 k) ![k+(m-j) / 2] !}
$$

and

$$
D_{m n}=\left(\frac{n !}{m !}\right)^{\frac{1}{2}} \beta^{m-n} e^{-\frac{1}{2}|\beta|^{2}} \mathcal{L}_{n}^{(m-n)}\left(|\beta|^{2}\right)
$$

where $\beta$ is given in Eq. $(2.5)$ and $\mathcal{L}_{n}^{(m-n)}\left(|\beta|^{2}\right)$ is the associate Laguerre polynomial. As we can see in Eq.(2.7), the coefficients of the SDNS are a sum of products of the coefficients of Displaced Number State and the Squeezed Number State. These coefficients $C_{j}$ are similar to those reported in Ref.[3], but defined here in a more simplified way. Computer manipulations involving the sum in Eq.(2.7) employs a truncation procedure, namely, $\sum_{j, m=0}^{\infty}(\ldots) \rightarrow \sum_{j, m=0}^{J, M}(\ldots)$. This procedure requires a rapid convergence of this series in the interval $\mathrm{j} \in[0, J]$ and $m \in[0, M]$, a property obeyed in our plots, with the condition that $\sum_{j=0}^{J} P(j)=1$.

Next, the photon number distribution corresponding to the SDNS and representing the probability of finding $j$ photons in the SDNS is easily obtained as 


$$
P(j) \equiv|\langle j \mid n, \alpha, z\rangle|^{2}=\left|\sum_{m=n}^{\infty} \frac{\sqrt{n ! j !\left(\frac{1}{2} \tanh r\right)^{(m-j) / 2}}}{(\cosh r)^{j+1 / 2}} \beta^{m-n} e^{-\frac{1}{2}|\beta|^{2}} \mathcal{L}_{n}^{(m-n)}\left(|\beta|^{2}\right) F(r, m, j) \cos ^{2}(j-m) \frac{\pi}{2}\right|^{2}
$$
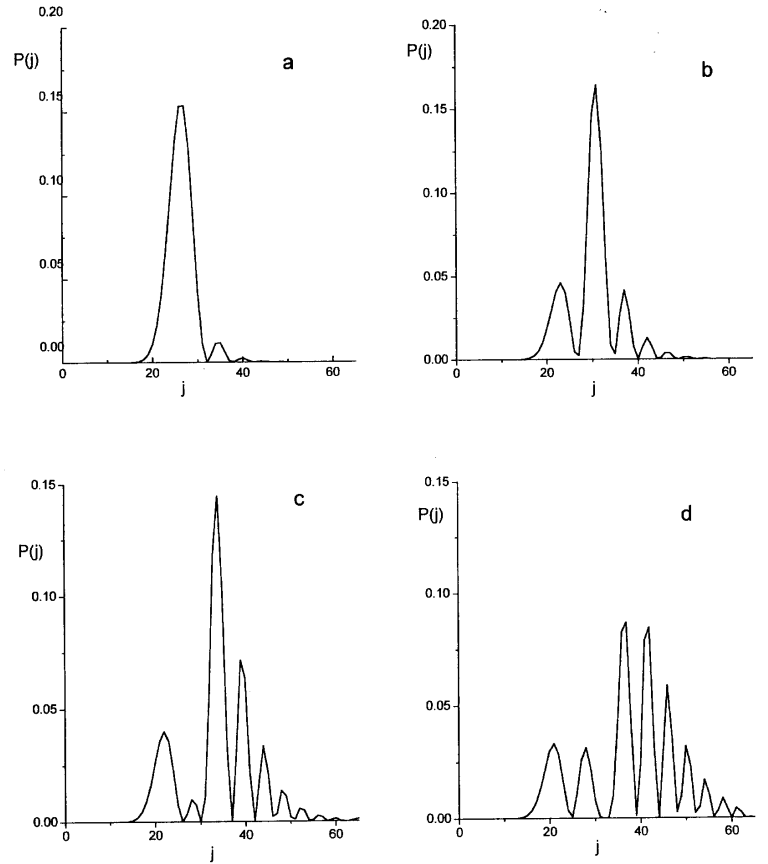

Figure 1. Photon number distribution $P(j)$ for the SDNS, for $r=1, \alpha=5, \theta=\phi=0$ and (a) $n=0$; (b) $n=1$; (c) $n=2 ;(\mathrm{d}) n=3$.

Fig. 1 shows plots of $P(j)$ as function of $j$, for $\alpha=5$, $r=1$ and $n=0,1,2,3$. Note that the distribution of photons shows oscillations which increase when $n$ increases. $P(j)$ is near zero around $j=32,38,42$ in Fig.(1a); $j=28,35,40$, 44 in Fig.(2b); $j=26,30,38$, 41, 43, 45, 47 in Fig.(1c) and $j=25,32,39,44,48$, 52 in Fig.(1d). These oscillations may be interpreted using the phase-space interference concept, introduced by Schleich and Wheeler [7]. According to Eq.(2.11) the photon number distribution $P(j)$ is given by the projection of the SDNS in the state $|j\rangle$. The pictoric representation of the states $|j\rangle$ and $|n, \alpha, z\rangle$ in the phase space is shown in Fig.(2), discussed below. When $n=0$ [Fig.1(a)], we obtain the Squeezed Coherent State [4], being one of the various particular cases of the SDNS. Other particular cases occur when $r=0, \alpha \neq 0$ and $\alpha=0, r \neq 0$ : in the first case, $\widehat{S}(z)$ given in Eq. $(2.3)$ becomes the unity and our SDNS coincides with the Displaced Number States [6], whose coefficients are given in Eq.(2.10); in the second case, $\widehat{D}(\alpha)$ given in Eq.(2.2) becomes the unity and the SDNS now coincides with the Squeezed Number State [5], whose coefficients are given in Eq.(2.8).

The elipses in Figs(2a) and (2b) represent the squeezed-number state and the SDNS, respectively, whereas the shadowed region in Fig.(2c) stands for the intersections among the SDNS (elipses) and the number states (circles). For example, the intersection between the SDNS and number state $|j\rangle$ gives the probability $P(j)$ to find $j$ photons in the SDNS. Interference comes from these intersections in the phase space, for this reason being named "interference in the phase space".

Next, the mean photon number and the photon number variance for the SDNS are given respectively by

$$
\langle\hat{j}\rangle=|\alpha|^{2}+(2 n+1) \sinh ^{2}(r)+n
$$

and

$$
\left\langle\Delta \hat{j}^{2}\right\rangle \equiv\left\langle\hat{j}^{2}\right\rangle-\langle\hat{j}\rangle^{2}=|\alpha|^{2}(2 n+1)\left[e^{2 r} \cos ^{2}\left(\theta-\frac{\phi}{2}\right)+e^{-2 r} \sin ^{2}\left(\theta-\frac{\phi}{2}\right)\right]+\left(n^{2}+n+1\right) 2 \sinh ^{2}(r) \cosh ^{2}(r) .
$$


Finding the Mandel's $Q$-parameter is now a straightforward calculation. We have,

$$
Q \equiv \frac{\left\langle\Delta \hat{j}^{2}\right\rangle-\langle\hat{j}\rangle}{\langle\hat{j}\rangle}=\frac{|\alpha|^{2}(2 n+1)\left[e^{2 r} \cos ^{2}\left(\theta-\frac{\varphi}{2}\right)+e^{-2 r} \sin ^{2}\left(\theta-\frac{\varphi}{2}\right)\right]+2 \sinh ^{2}(r) \cosh ^{2}(r)\left(n^{2}+n+1\right)}{|\alpha|^{2}+\sinh ^{2}(r)(2 n+1)+n}-1
$$

which gives the departure from the Poissonian statistics: for positive, zero or negative values of $Q$ the statistics are super-Poissonian, Poissonian or sub-Poissonian, respectively. For $n=0$ it corresponds to the squeezed coherent state [3], in which the statistics is sub or superPoissonian, depending on the values of parameters $\theta, \varphi$ and $r$.

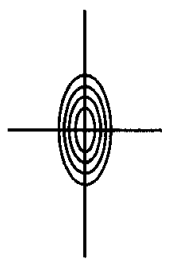

a)

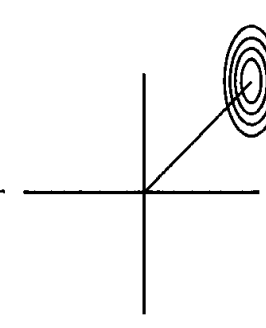

b)

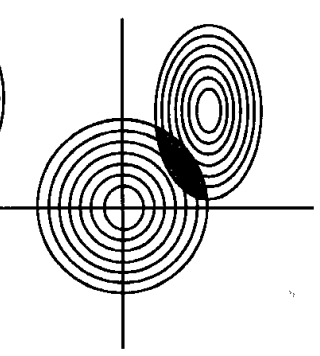

c)
Figure 2. Pictoric representation of: (a) squeezed number states, (b) squeezed displaced number states, and (c) intersection of squeezed displaced number states and number states.

Fig.(3) shows plots of Mandel's $Q$ - parameter as function of $r$, for $\alpha=5, \theta=\varphi=0$ and $n=0,1,2,3$. When $n=0$, for negative, zero and positive values of the squeezing parameter $r$, we have that $Q<0, Q=0$ and $Q>0$, and it is sub-Poissonian, Poissonian and super-Poissonian, respectively. When $n \geq 1$, the statis- tics becomes different from that for squeezed coherent states and the sub-Poissonian effect disappears..

The variances $\Delta \hat{X}_{1}$ and $\Delta \hat{X}_{2}$ of the quadrature operators $\widehat{X}_{1}$ and $\widehat{X}_{2}$, when calculated in the SDNS, result respectively ( with $\Delta \widehat{X}_{i}^{2}=\left\langle\widehat{X}_{i}^{2}\right\rangle-\left\langle\widehat{X}_{i}\right\rangle^{2}, i=1,2$ ),

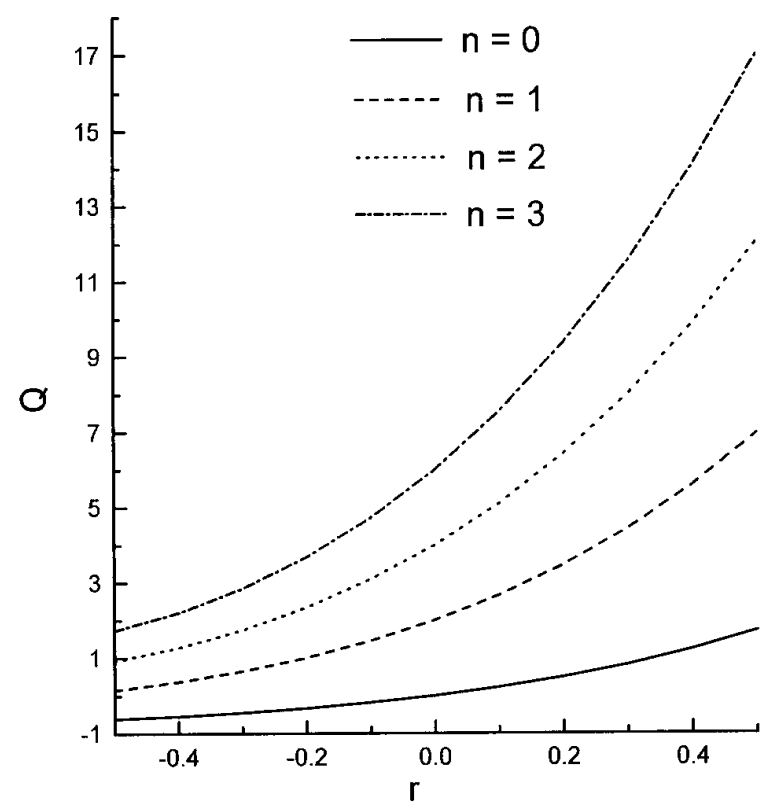

Figure 3. Mandel's $Q$-parameter as a function of $r$ for the SDNS, for $\theta=\phi=0, \alpha=5$ and (a) $n=0$; (b) $n=1$; (c) $n=2$; (d) $n=3$.

$$
\begin{aligned}
& \Delta \hat{X}_{1}^{2}=\frac{1}{4}\left[e^{2 r} \cos ^{2} \frac{\phi}{2}+e^{-2 r} \sin ^{2} \frac{\phi}{2}\right]+n\left[e^{2 r} \cos ^{2} \frac{\phi}{2}+e^{-2 r} \sin ^{2} \frac{\phi}{2}\right] \\
& \Delta \hat{X}_{2}^{2}=\frac{1}{4}\left[e^{2 r} \sin ^{2} \frac{\phi}{2}+e^{-2 r} \cos ^{2} \frac{\phi}{2}\right]+n\left[e^{2 r} \sin ^{2} \frac{\phi}{2}+e^{-2 r} \cos ^{2} \frac{\phi}{2}\right]
\end{aligned}
$$

Squeezing occurs when $\Delta \hat{X}_{i}<\frac{1}{2}, i=1$ or 2 . Depending on the signal of the squeeze parameter $r$, the effect occurs in the quadrature $\Delta \widehat{X}_{1}$ or $\Delta \widehat{X}_{2}$.
Fig.(4) shows plots of $\Delta \widehat{X}_{1}^{2}$ as function of $r$, for $\alpha=5, \theta=\phi=0$ and $n=0,1,2,3$. For $n=0$, 
$\Delta \widehat{X}_{1}^{2}$ shows squeezing for negative values of $r$ (the opposite occurs for $\Delta \widehat{X}_{2}^{2}$ as we can see by comparing the Eqs.(2.15) and (2.16)). When $n$ increases the squeezing effect disappears.

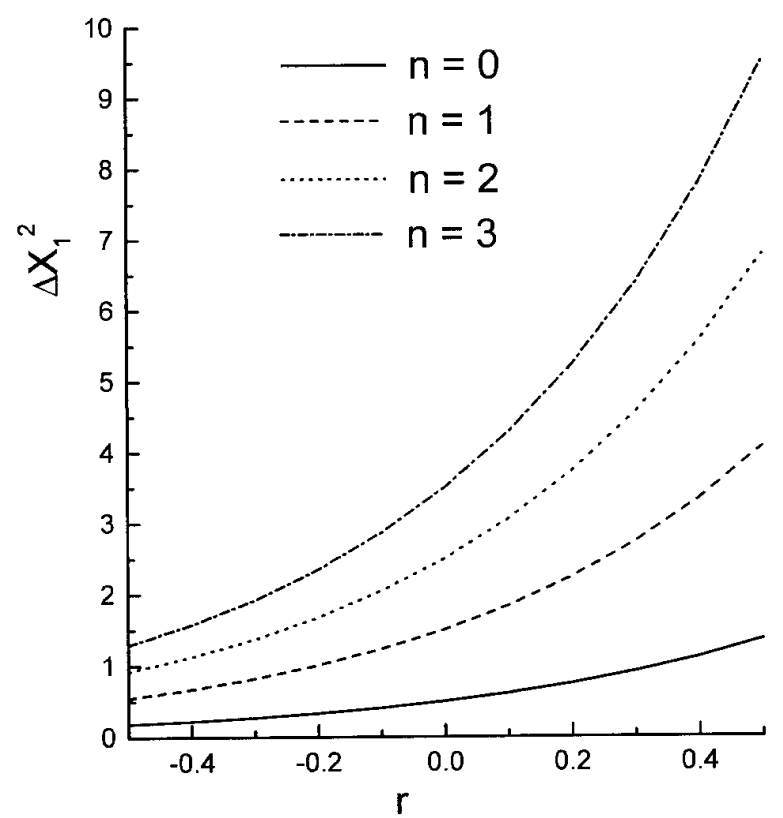

Figure 4. Variance in quadratures $\Delta \hat{X}_{i}$ as a function of $r$ for the SDNS, for $\phi=0$ and (a) $n=0$; (b) $n=1$; (c) $n=2$; (d) $n=3$.

\section{Photon-Counting Distribu- tion}

The probability $G(T)$ of detecting no photons in an arbitrary state, with photon number distribution $P(j)$, is given by [8]

$$
\begin{gathered}
G(T)=\sum_{r=0}^{\infty} \frac{(-\eta \mu T)^{r}}{r !} \sum_{j=0}^{\infty}\left\langle j\left|\hat{a}^{+r} a^{r}\right| j\right\rangle P(j) \\
=\sum_{j=0}^{\infty} P(j)[1-\eta \mu T]^{j}
\end{gathered}
$$

valid for small counting-time separation between successive photodetections, where $\eta$ is the efficiency of the detector. The quasimonochromatic field at the detector is described by the photon-flux operator $\widehat{I}(t)$ and $\mu$ is a factor converting the photon number operator $\hat{j}=\hat{a}^{+} \hat{a}$ into a photon flux operator $\hat{I}$ and the mean photoelectron counting rate is $\eta\langle\widehat{I}(t)\rangle$. For a small time intervals $(\eta \mu T<<1)$ we can write:

$$
\int_{0}^{T} \widehat{I}(t) d t=\hat{I} T=\mu T \hat{n}
$$

By introducing an adjustment parameter $s$ in the Eq.(3.1), in the following manner:

$$
G(T, s)=\sum_{j=0}^{\infty} P(j)[1-s \eta \mu T]^{j}
$$

we can calculate the photon-counting distribution $P(m, T)$, which gives the probability of detecting $m$ photons in the (small) interval $[0-T]$, according to Ref.[8]:

$$
\begin{aligned}
& P(m, T)=\left.\frac{(-1)^{m}}{m !} \frac{d^{m}}{d s^{m}} G(s, T)\right|_{s=1} \\
= & \sum_{j=m}^{\infty} \frac{j !}{m !(j-m) !}(\gamma T)^{m}[1-\gamma T]^{j-m} P(j),
\end{aligned}
$$

where we put $\gamma=\eta \mu$. It coincides with the photonnumber distribution $P(j)$, when quantum efficiency is unity, $\gamma T=1$. In practice quantum efficiency is less than unity and the photon-counting distribution is only related to $P(j)$, as in Eq. $(3.4)$.

Fig.(5) shows plots of $P(m, T)$ as function of $m$ for $r=1, \alpha=5, \theta=\varphi=0, \mu T=0.8$ and various values of $n$. Note that it differs from the photon number distribution $P(j)$ (dashed curve). The difference between them increases when $n$ increases, as can be seen in Figs.(5a)-(5d).

Fig.(6) Same as in Fig.(5), for $\alpha=5, r=1$ and $n=1$ and various values of quantum efficiency $\gamma T$. Note again that $P(m, T)$ differs from $P(j)$, this difference disappearing when $\gamma T \rightarrow 1$ (ideal photodetection). 

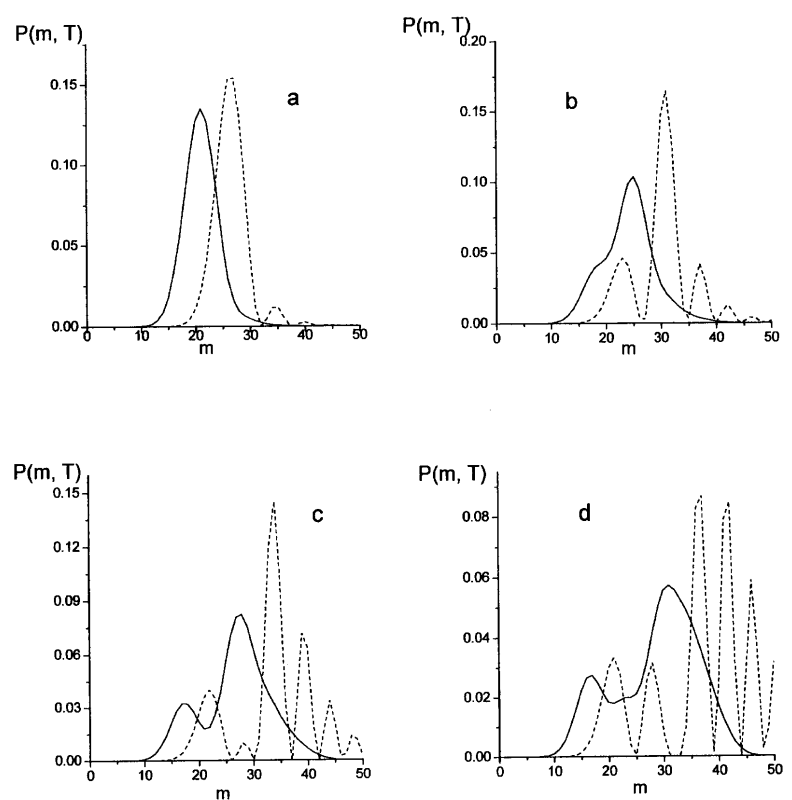

Figure 5. Photon-counting distribution $P(m, T)$ for the SDNS, for $r=1, \alpha=5, \theta=\phi=0, \mu T=0.8$ and (a) $n=0$, (b) $n=1$, (c) $n=2$, (d) $n=3$, in comparison with the photon number distribution $P(j)$ (dashed curves).
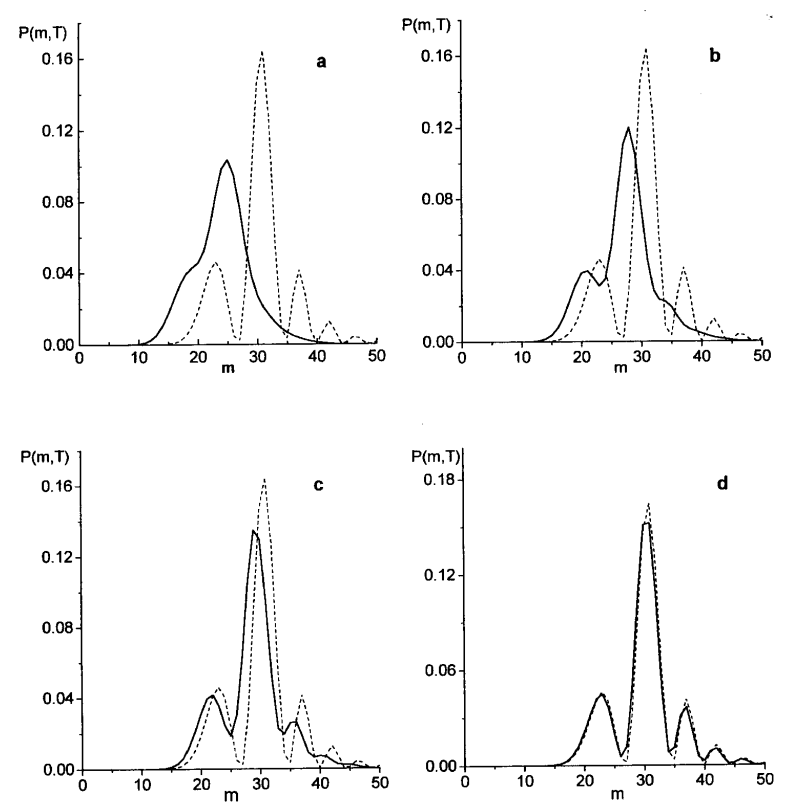

Figure 6. Same as in Fig.(4), for $r=1, \alpha=5, \theta=\phi=0$, $n=1$ and (a) $\mu T=0.8$ (b) $\mu T=0.9$, (c) $\mu T=0.95$, (d) $\mu T=0.99$, in comparison with the photon number distribution $P(j)$ (dashed curves).

\section{Waiting-Time Distribution}

The waiting-time distribution $\omega(T)$, which denotes the probability of detecting the next photon at time $T$, given that a photon was detected at time $T=0$, can be written in terms of $G(\tau)$ given in Eq.( 3.1$)$. We have [8],

$$
\omega(T)=\frac{1}{\eta\langle\hat{I}\rangle} \frac{d^{2} G(T)}{d T^{2}}
$$

$$
=\frac{\gamma}{\eta} \sum_{j=2}^{\infty} \frac{j(j-1)}{\langle\hat{j}\rangle^{2}}(1-\gamma T)^{j-2} P(j)
$$

where $\eta$ and $\gamma$ concern to the quantum efficiency. Using a scaled time defined by $\tau=\gamma T\langle\hat{j}\rangle$, and considering $\eta=\gamma=1$, we can write the Eq.(4.1) in the form

$$
\omega(\tau)=\sum_{j=2}^{\infty} \frac{j(j-1)}{\langle\hat{j}\rangle^{2}}\left(1-\frac{\tau}{\langle\hat{j}\rangle}\right)^{j-2} P(j) .
$$

So, the photon sequence in time can be written in terms of $\omega(\tau)$ [8]. For a coherent state, the waiting-time distribution $\omega(\tau)$ results

$$
\omega_{c}(\tau)=e^{-\tau}
$$

which comes from the substitution of the photonnumber distribution $P(j)$ for a coherent state, given by

$$
P(j)=e^{-|\alpha|^{2}} \frac{|\alpha|^{2 j}}{j !},
$$

in Eq.(4.2), with subsequent evaluation of the sum. For $\tau=0$, we have $\omega_{c}(0)=1$. So, when $\omega(0)<1$ the light field has a photon sequence antibunching, whereas for $\omega(0)>1$ it shows a photon sequence bunching. Considering a field in the SDNS and substituting its

photon-number distribution given by Eq.(2.11), in Eq.(4.2), we can evaluate numerically this sum to obtain the waiting-time distribution $\omega(\tau)$ for the SDNS. 

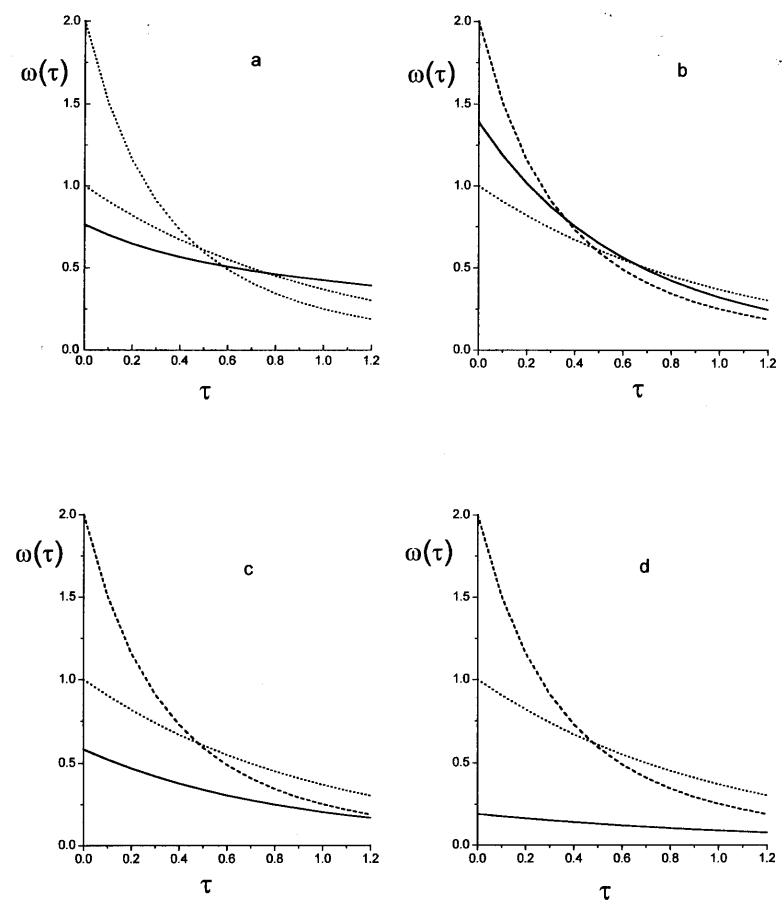

Figure 7. Waiting-time distribution $\omega(\tau)$ for the SDNS as a function of $\tau$, for $\alpha=1, \theta=\phi=0$ and (a) $r=0,4 ; n=0$ (b) $r=1 ; n=3$ (c) $r=2 ; n=1$ (d) $r=2 ; n=2$, in comparison with $\omega(\tau)$ of the coherent state (dashed curves) [cf. Eq.(4.3)] and thermal state (dotted curves) [cf. Eq.(4.5)].

Fig.(7) shows the waiting-time distribution $\omega(\tau)$ as function of $\tau$, for $\alpha=1, \theta=\varphi=0$ and different values of $r$ and $n$ characterizing the SDNS (solid curves). Note that depending on the parameters $r$ and $n$, the SDNS shows antibunching effect, $\omega(0)<1$, as we can see in Figs.(7a), (7c) and (7d), and also bunching effect, $\omega(0)>1$, shown in Fig.(7b). For comparison, we have also plotted $\omega(\tau)$ for a coherent state (dashed curves), given in Eq.(4.3), and thermal field (dotted curves), given by [8]

$$
\omega(\tau)=\frac{2}{(1+\tau)^{3}}
$$

\section{Conclusion}

We have studied statistical properties of the Squeezed Displaced Number State. It was shown that the photon number distribution of SDNS is a sum of the product of the coefficients of Displaced Number State and the Squeezed Number State [cf. Eq.(2.7)], resulting in one of them in appropriated limits. The photon-counting distribution $P(m, T)$ and waiting-time distribution $\omega(\tau)$ were also analyzed. The antibunching effect, which measures photon sequence in time, was analyzed in terms of $\omega(\tau)$. We have shown that, for convenient choice on parameters distinguishing the SDNS from others in the literature, the photon-number distribution $P(j)$ exhibits oscillations which are stronger than those found in particular states obtained in this scheme, as mentioned before. Concerning to some aspects [See Eqs.(2.7)-(2.10)] the present approach simplifies considerably the calculations, in comparison with others in the literature [3]. Finally, it is worth stressing that since the SDNS contains various particular states studied in the literature (e.g., number state, coherent state, squeezed number state, displaced number state, etc.), then it permits an unified approach incorporating all these states, and their properties, as special cases. So, it constitutes a theoretical tool allowing us to get a compact treatment including various important states of the literature, as well as furnishing a new interpolating state [9] alternative to the binomial state by Stoler et al.[10]. Concerning to its generation, a method first suggested by Glauber [11] and Louisell [12], when applied to a field initially in a number state $|n\rangle$ leads it to a displaced-number state $|\alpha, n\rangle[13]$. Subsequent squeezing generated by the variation of a cavity-parameter [14] would lead the state $|\alpha, n\rangle$ to the SDNS. Alternative procedure, generating similar states can be seen in Ref.[15].

\section{Acknowledgments}

This research was partially supported by Conselho Nacional de Desenvolvimento Científico e Tecnológico (CNPq), of Brazil, and PRONEX/MCT/FINEP.

\section{References}

[1] Collection of works on squeezing can be found in: J. Opt. Soc. Am. B 4, (10) (1987).

[2] C. M. Caves, Phys. Rev. D 23, 921 (1981).

[3] P. Král, J. of. Mod. Opt. 37, 889 (1990).

[4] H. P. Yuen, Phys. Rev. A 13, 2226 (1976).

[5] M. S. Kim, F. A. M. de Oliveira and P. L. Knight, Phys. Rev. A 40, 2494 (1989).

[6] F. A. M. de Oliveira, M. S. Kim and P. L. Knight, Phys. Rev. A 41, 2645 (1990).

[7] W. Schleich and J. A. Wheeler, J. Op. Soc. Am. B 4, 1715 (1987); W. Schleich, D. F. Walls and J. A. Wheeler, Phys. Rev. A 13, 2226 (1976). 
[8] Reeta Vyas and Surendra Singh, Phys. Rev. A 38, 2423 (1988); H. J. Carmichael, Surendra Singh, Reeta Vyas and P. R. Rice, Phys. Rev A 39, 1200 (1989); B. E. A. Saleh, Photoelectron Statistics (Springer-Verlag, Berlin (1978)).

[9] Célia M. A. Dantas, B. Baseia and V. S. Bagnato, Phys. Scripta 58, 145 (1998).

[10] D. Stoler, B. E. A. Saleh and M. C. Teich, Opt. Acta 32, 345 (1985).

[11] R. J. Glauber, Phys. Rev. 131, 2766 (1963); see also: H. M. Nussenzveig, Introduction to Quantum Optics (Gordon and Breach, NY (1973)), p.73.

[12] W. H. Louisell, Quantum Statistical Properties of Radiation (Wiley\&Sons, NY (1973)), p. 231.
[13] For the generation of the particular case $|\alpha, 0\rangle$, see: M. Brune, S. Haroche, J. M. Raimond, L. Davidovich and N. Zagury, Phys.Rev. A45, 5193 (1992); L. Davidovich, A. Maali, M. Brune, J. M. Raimond and S. Haroche, Phys. Rev. Lett. 71, 2360 (1993); S. Zheng and G.Guo, Opt. Commun. 133, 139 (1997).

[14] See, e.g., A. D. Janussis and B.S. Bartzis, Phys. Lett. A129, 263 (1988); C. F. Lo, N. Cim. B105, 497 (1990); Phys. Rev. A43, 404 (1991); B. Baseia et al. Phys. Rev. A45, 5308 (1992); A46, 5885 (1992); A45, 1320 (1992); Phys. Lett. A170, 311 (1992); F. li Li, Phys. Lett. A168, 400 (1992); T. Kiss, P. Adam and J. Janszky, Phys. Lett. A192, 311 (1994).

[15] H. Moya-Cessa, J. Mod. Opt. 42, 1741 (1995). 